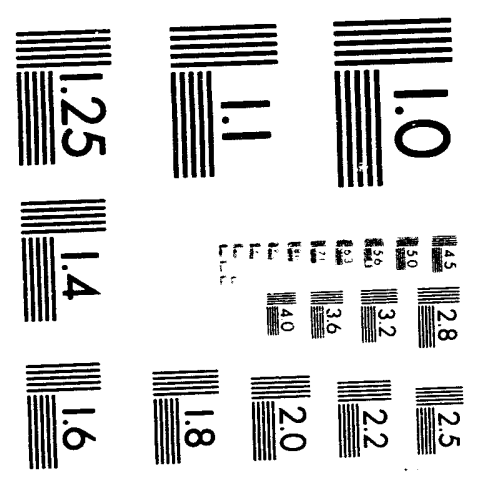



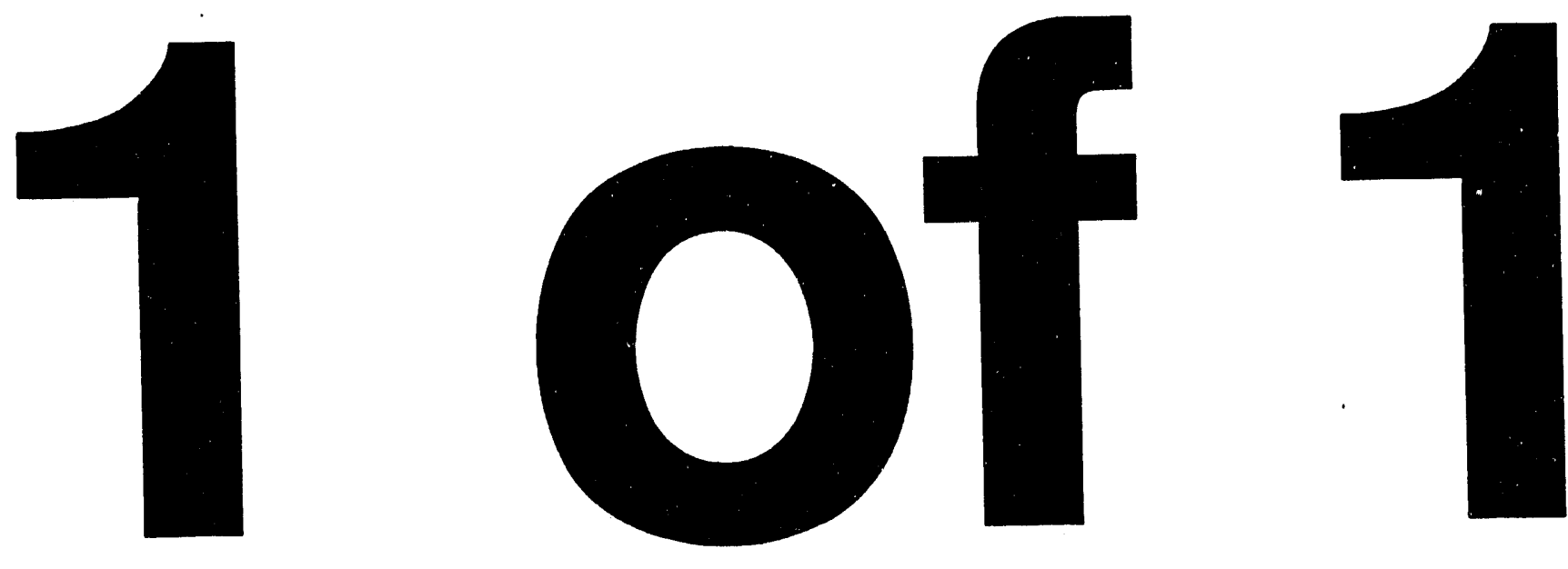


\section{INITIAL WEIGHTS AND DIMENSIONS OF CORROSION COUPONS INSTALLED IN IDMS IN AUGUST 1993 (U)}

by

K. Imrick

Westinghouse Savannah River Company

Savannah River Site

Aiken, South Carolina 29808

This paper was prepared in connection with work done under the above contract number with the U. S.

Department of Energy. By acceptance of this paper, the publisher and/or recipient acknowledges the U. S. Government's right to retain a nonexclusive, royalty-free license in and to any copyright covering this paper, along with the right to reproduce and to authorize others to reproduce all or part of the copyrighted paper. 


\section{DISCLAIMER}

This report was prepared as an account of work sponsored by an agency of the United States Government. Neither the United States Government nor any agency thereof, nor any of their employees, makes any warranty, express or implied, or assumes any legal liability or responsibility for the accuracy, completeness, or usefulness of any information, apparatus, product, or process disclosed, or represents that its use would not infringe privately owned rights. Reference herein to any specific commercial product, process, or service by trade name, trademark, manufacturer, or otherwise does not necessarily constitute or imply its endorsement, recommendation, or favoring by the United States Government or any agency thereof. The views and opinions of authors expressed herein do not necessarily state or reflect those of the United States Government or any agency thereof.

This report has been reproduced directly from the best available copy.

Available to DOE and DOE contractors from the Office of Scientific and Technical Information, P. O. Box 62, Oak Ridge, TN 37831; prices available from (615) $576-8401$.

Available to the public from the National Technical Informa ory. U. Department of Commerce, 5285 Port Royal Rd., Springfield, V 32501 
CC: J.R. Zamecnik, 704-T

L.F. Landon, 704-T

R.A. Jacobs, 704-T

R.E. Eibling, 704-T

D.A. Crowley, 704-1T

C.T. Randall, 704-T

N.H. Kuehn, 704-1T

J.C. Marek, 704-T

R. Minichan, 704-T

J.F. Ortaldo, 704-S

W.D. Kimball, 704-S

J.A. Gentilucci, 704-S

H.H. Elder, 704-S

J.T. Gee, 704-S

D.C. Iverson, 704-2S

J.T. Carter, 704-S

E.W. Holtzscheiter, 773-A

M.J. Plodinec, 773-A

D.F. Bickford, 773-A

J.D. Cohen, 730-A

T.L. Capeletti, 773-A

R.L. Bickford, 730-A

C.F. Jenkins, 730-A

N.C. Iyer, 773-A

G.T. Chandler, 773-A

PIM, 703-43A (4)

MTS Files, 773-A 


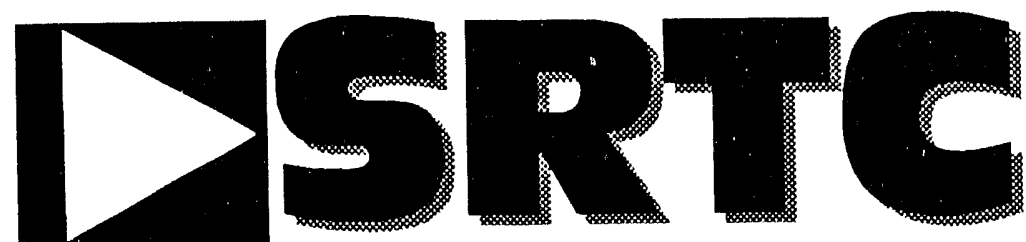

Savannah River Technology center

October 4, 1993

To: J. R. Zamecnik

Defense Waste Processing Technology

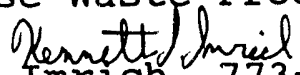

From: K. J. Imrich, 773-A

Materials Technology section

\section{BUBJECT DESCRIBERS}

IDMS

Corrosion

Coupon

Documentation

T-Area

DWPF

Retention: Lifetime WSRC-TR-93-518

\section{Bune \& Masema}

Bruce Wiersma, Technical Reviewer,

Mat. Appl. \& Corr. Tech.

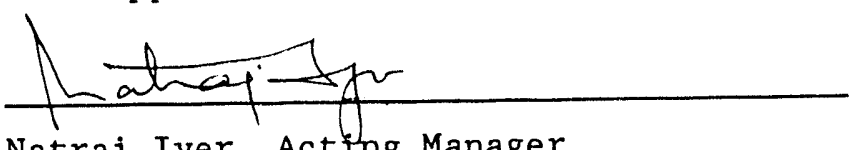

Natraj Iyer, Acting Manager,

Mat. App1. \& Corr. Tech.

\section{INITIAL WEIGHTS AND DIMENSIONS OF CORROSION COUPONS INSTALLED IN IDMS IN AUGUST 1993 (U)}

\section{summary}

New corrosion coupons were installed in the Feed Preparation system and the Melter/Off-Gas System of Integrated DWPF Melter system (IDMS) in August 1993. The new coupons are replacements for similar coupons which were in the IDMS since 1989 but were removed for metallurgical evaluation in April 1993. Original coupons still remain on the corrosion racks in the Melter and the off-Gas stack. Baseline data for the replacement coupons are described in this report.

\section{Background}

New corrosion coupons were installed in the IDMS in August 1993 as part of the continuing IDMS Materials Evaluation Program. This program was establish to confirm the performance of Defense Waste Processing Facility (DWPF) reference materials of construction and other alternate materials. The new coupons are replacements for similar coupons which were in the IDMS since 1989 but were removed for metallurgical evaluation in April 19932,3. Coupons on the racks in the Melter and the off-Gas stack were not replaced. Therefore, these racks still contain the original coupons which were installed in 1989. 
J. R. Zamecnik

October 4, 1992

Page 2 of 18

WSRC-TR-93-518

Replacement coupons were installed throughout the IDMS in both the Feed Preparation and the and Melter/Off-Gas systems and consisted of standard flat and U-bend specimens. Some of the coupons were welded with matching filler metal and are designated with a " $W$ " in the coupon identification number. In order to evaluate the effect of crevice corrosion, crevice washers were used on most specimens. The following list contains the locations (see Figure 1) of the thirteen corrosion racks which were assembled and placed back into the IDMS:

Feed Preparation system

1a) Slurry Receipt Adjustment Tank and Slurry Mix Evaporator (SRAT/SME) - Vapor Space,

1b) SRAT/SME - Liquid/Vapor Zone,

2) SRAT/SME Condenser - Vapor Inlet,

3) Mercury Water Wash Tank (MWWT) - Vapor Space,

4a) Melter Feed Tank - Vapor Space,

4b) Melter Feed Tank - Liquid/Vapor Zone,

Melter/Off-Gas System

5) Quencher - Inlet,

6) Quencher - outlet,

7a) Off-Gas Condensate Tank (OGCT) - Vapor space,

7b) Off-Gas Condensate Tank - Liquid Zone,

8) Off-Gas Condenser - Vapor Inlet,

9) Off-Gas Condenser - Drain,

10) Steam Atomized Scrubber (SAS) - Drain Line,

11) High Efficiency Mist Eliminator (HEME) - Inlet,

12) HEME - outlet, and

13) High Efficiency Particulate Air (HEPA) Filter - Inlet.

\section{Coupon Documentation}

Descriptions of the coupons are presented in this report on a rack by rack basis and include the material type, dimensions, weight, and orientation of the replacement corrosion coupons. Table 1 contains the material compositions. Coupons identified with a "NM" indicate that the coupon has no markings on the coupon other than the identification number and alloy type. Coupons identified with IDMS were extra original coupons that were purchased before 1989 but were never installed in the IDMS. Coupons identified as "NEW" were purchased in 1993 and are marked with alloy type, identification number, and heat. number. Documentation of the assembled corrosion racks is contained in Tables 2 through 14 . 
J. R. Zamecnik

October 4, 1992

Page 3 of 18

WSRC-TR-93-518

\section{References}

1. S. M. Nordwick and J. R. Zamecnik, DWPT Task Plan IDMs Materials Inspections, DWPTQA-89-0042, Savannah River Site, Aiken, SC 29802 (December 12, 1989).

2. S. M. Nordwick, Equipment Engineering Division Area Metallurgical Report, IDMS corrosion Coupons, EED890385, Savannah River Site, Aiken, SC 29802 (March 27, 1989).

3. K. J. Imrich and C. F. Jenkins, Final Examination of IDMs corrosion Coupons (U), WSRC-TR-93-461, Savannah River Site, Aiken, SC 29802 (September 16, 1993). 
J. R. Zamecnik

October 4, 1992

Page 4 of 18

WSRC-TR-93-518

\section{FIGURE 1}

schematic of IDMs showing Locations of Corrosion Coupons And The Materials of construction of The various process vessels

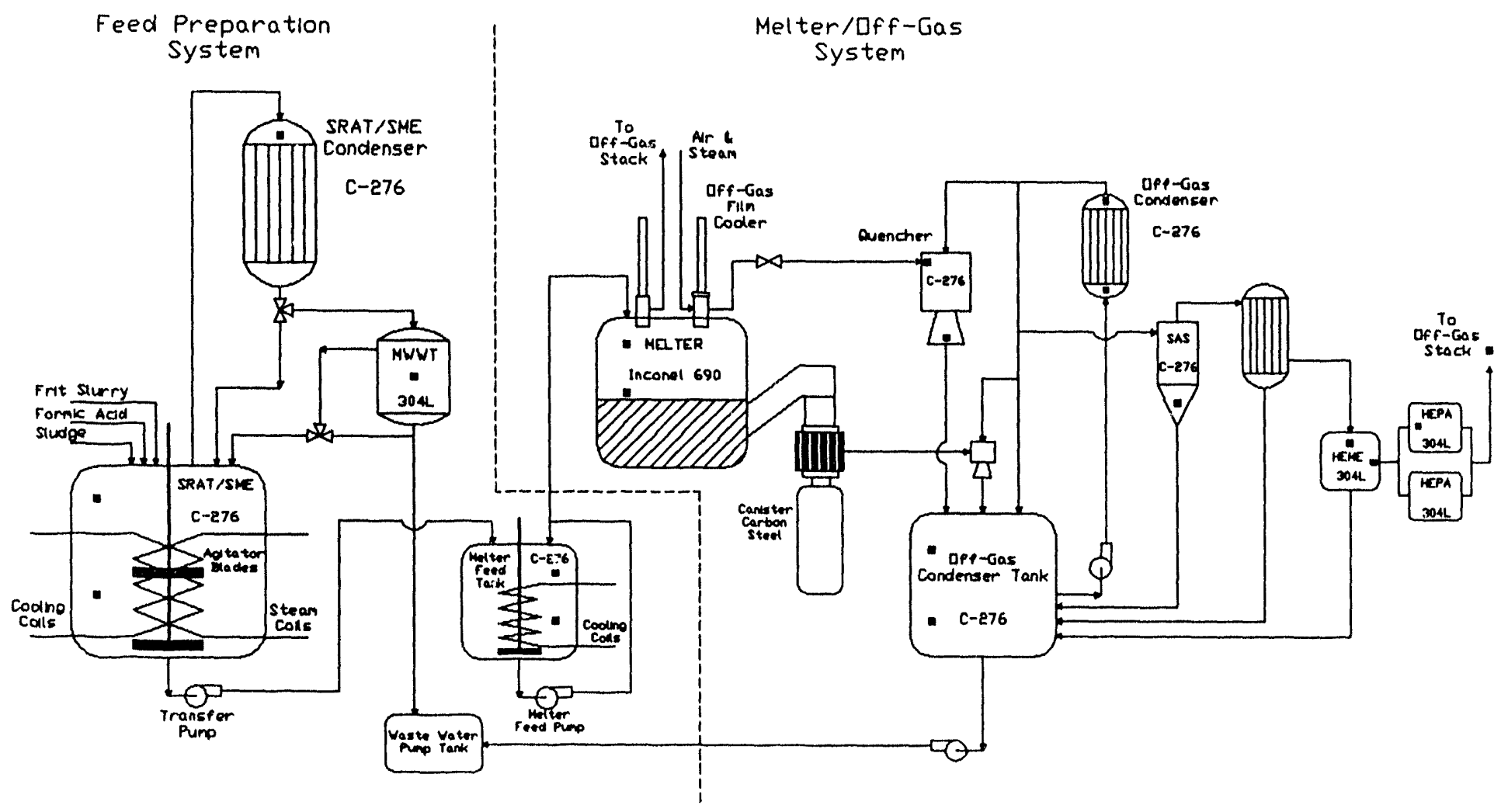

- Corrosion Rack Location 
J. R. Zamecnik

October 4, 1992

Page 5 of 18

WSRC-TR-93-518

Table 1

Material Compositions

\begin{tabular}{|c|c|c|c|c|c|c|c|c|c|c|c|c|c|c|c|}
\hline Manufarturer & Material & Heat No. & Marking & $\mathrm{Ni}$ & $\mathrm{Cr}$ & $\mathrm{Fe}$ & $W$ & Co & Mo & $M n$ & Si & $\mathbf{P}$ & $S$ & $\mathrm{C}$ & Other \\
\hline J\&L Speciality & $304 \mathrm{~L}$ & E673 & IDMS & 8.83 & 18.23 & 69.79 & & 0.10 & 0.24 & 1.79 & 0.61 & 0.030 & 0.018 & 0.026 & N $0.086, \mathrm{Cu} 0.250$ \\
\hline Allegheny-Ludlum & $304 \mathrm{~L}$ & $\mathrm{~J} 160$ & New & 8.11 & 18.26 & 70.18 & & & 0.30 & 1.86 & 0.56 & 0.320 & 0.002 & 0.020 & Cu $0.30, N 0.09$ \\
\hline Allegheny-Ludlum & $316 \mathrm{~L}$ & E616 & IDMS & 10.12 & 16.22 & 68.80 & & 0.10 & 2.12 & 1.70 & 0.60 & 0.033 & 0.001 & 0.020 & $\mathrm{~N} 0.040 . \mathrm{Cu} 0.250$ \\
\hline Allegheny-Ludlum & $316 \mathrm{~L}$ & $\mathrm{H} 980$ & New & 10.18 & 16.30 & 68.91 & & & 2.13 & 1.90 & 0.50 & 0.030 & 0.001 & 0.019 & N 0.03 \\
\hline Eastern Stainless & $317 \mathrm{~L}$ & $E 309$ & IDMS & 14.00 & 18.29 & 61.75 & & 0.20 & 3.13 & 1.63 & 0.59 & 0.026 & 0.011 & 0.017 & $\mathrm{~N} 0.072, \mathrm{Cu} 0.280$ \\
\hline Tull Metals & $317 L$ & $\mathrm{H} 708$ & New & 13.23 & 18.19 & 62.47 & & 0.15 & 3.11 & 1.78 & 0.55 & 0.031 & 0.001 & 0.019 & $\mathrm{Cu} 0.42, \mathrm{~N} 0.047$ \\
\hline Allegheny-Ludlum & AL6XN & E881 & IDMS & 23.92 & 20.67 & 47.80 & & & 6.32 & 0.46 & 0.30 & 0.028 & 0.001 & 0.026 & $\mathrm{~N} 0.230 . \mathrm{Cu} 0.250$ \\
\hline Haynes & G-3 & E788 & IDMS & 44.11 & 22.46 & 19.84 & 0.80 & $1 . \pi$ & 7.49 & 0.77 & 0.49 & 0.019 & 0.002 & 0.005 & Cu $1.880, \mathrm{Cb}+\mathrm{Ta} 0.360$ \\
\hline INCO & 674 & G620 & & 53.92 & 45.50 & 0.13 & & & & 0.02 & 0.09 & & 0.003 & 0.050 & $\mathrm{Ti} \quad 0.290$ \\
\hline Haynes & $\mathrm{C}-22$ & $\mathrm{~F} 120$ & IDMS & 56.71 & 21.10 & 4.10 & 2.90 & 0.93 & 13.80 & 0.28 & 0.03 & 0.008 & 0.002 & 0.002 & $\vee 0.140$ \\
\hline Haynes & $\mathrm{C}-22$ & $\mathrm{H} 157$ & New & 56.34 & 21.30 & 4.40 & 2.90 & 1.14 & 13.40 & 0.29 & 0.05 & 0.010 & $0.0 \mathrm{c} 2$ & 0.004 & $\vee 0.170$ \\
\hline Haynes & C-276 & F266 & IDMS & 57.26 & 15.54 & 5.77 & 3.80 & 1.33 & 15.63 & 0.45 & 0.03 & 0.015 & 0.002 & 0.003 & $v 0.170$ \\
\hline Haynes & C.276 & $J 018$ & New & 58.67 & 15.26 & 5.35 & 3.34 & 1.35 & 15.39 & 0.48 & 0.02 & 0.007 & 0.002 & 0.003 & $\vee 0.130$ \\
\hline Teledyne-Allvac & Allcorr & D352 & IDMS & 57.99 & 30.93 & 0.11 & & 0.01 & 9.97 & 0.01 & 0.02 & 0.005 & 0.001 & 0.030 & $\begin{array}{c}\text { Al } 0.230,80.002 \\
\text { Zr } 0.010, \vee 0.010, \mathrm{Cb}+\mathrm{Ta} \\
0.420, \mathrm{Ti} 0.240 \\
\end{array}$ \\
\hline INCO & 690 & $\operatorname{cos4}$ & IDMS & 59.40 & 29.10 & 10.48 & & & & 0.42 & 0.30 & & 0.003 & 0.010 & $\mathrm{Ti} 0.290$ \\
\hline INCO & 625 & E340 & IDMS & 60.87 & 21.14 & 4.49 & & & 8.52 & 0.06 & 0.06 & 0.004 & 0.001 & 0.010 & $\begin{array}{c}\mathrm{Cb}+\mathrm{Ta} \text { 3.40. Ti } 0.240 \\
\text { Al } 0.20\end{array}$ \\
\hline Duriron & CW7M & E164 & New & 61.52 & 18.79 & 1.09 & & & 17.51 & 0.59 & 0.48 & 0.007 & 0.005 & 0.009 & \\
\hline Haynes & 556 & $\mathrm{H} 148$ & New & 21.82 & 21.78 & 31.35 & 2.20 & 18.25 & 2.92 & 0.97 & 0.41 & 0.009 & 0.002 & 0.100 & Al 0.190, B 0.002 \\
\hline Haynes & 8727 & No Heat No. & Old No Marking & 42.49 & 28.76 & 2.31 & 0.10 & 24.35 & 0.05 & 0.38 & 1.50 & 0.005 & 0.001 & 0.057 & \\
\hline Haynes & 25 & D823 & IDMS & 9.84 & 20.25 & 2.25 & 15.15 & 50.39 & 0.34 & 1.45 & 0.21 & 0.005 & 0.002 & 0.110 & \\
\hline Stellite (Cast) & ST6 & F905 & New & 2.62 & 27.63 & 2.92 & 4.07 & 59.58 & 0.41 & 0.27 & 1.07 & & & 1.430 & \\
\hline Stellite (Wrought) & $6 B$ & $\sqrt{256}$ & New & 2.29 & 30.25 & 0.87 & 3.78 & 58.34 & 0.91 & 1.39 & 1.07 & & & 1.100 & \\
\hline Stellite & 6BLC & No Heat No. & Old No Marking & 2.73 & 30.35 & 0.10 & 3.98 & 60.35 & 0.10 & 1.70 & 0.58 & 0.011 & 0.004 & 0.100 & \\
\hline
\end{tabular}

\begin{tabular}{|c|c|c|c|c|c|c|c|c|c|}
\hline Manufacturer & Material & $\mathrm{Al} 2 \mathrm{O} 3$ & SiO2 & MgO & $\mathrm{Na2O}$ & $\mathrm{CaO}$ & Fe2O3 & $\mathrm{Cr} 203$ & $\mathbf{K} 20$ \\
\hline Bolt Technical Ceramics & Alumina & 99.70 & 0.11 & 0.22 & 0.008 & 0.005 & 0.060 & 0.007 & 0.00 \\
\hline
\end{tabular}

- (IDMS) = Coupons purchased before 1989 and marked with alloy, coupon number and IDMS.

- (OLD NM) = Coupons purchased in 1992 and marked with only alloy and coupon numbers.

- $(\mathrm{New})=$ Coupons purchased May, 1993 and marked with alloy, heat no. and coupon number. 
J. R. Zamecnik

October 4, 1992

Page 6 of 18

WSRC-TR-93-518

Table 2

SRAT/SME - Vapor space and Liquid/Vapor zone

\begin{tabular}{|c|c|c|c|c|c|c|c|}
\hline $\begin{array}{c}\text { Coupon } \\
\text { Location }\end{array}$ & $\begin{array}{c}\text { Material } \\
\text { Type }\end{array}$ & $\begin{array}{c}\text { Coupon } \\
\text { No. }\end{array}$ & $\begin{array}{c}\text { Weight } \\
(\mathrm{g})\end{array}$ & $\begin{array}{c}\text { Length } \\
\text { (in) }\end{array}$ & $\begin{array}{c}\text { Width } \\
\text { (in) }\end{array}$ & $\begin{array}{c}\text { Hole Dia. } \\
\text { (in) }\end{array}$ & $\begin{array}{c}\text { Thickness } \\
\text { (in) }\end{array}$ \\
\hline Vapor & HC22W & 8 (NEW) & 23.0491 & 1.9871 & 0.7511 & 0.3742 & 0.1208 \\
\hline Vapor & HC276W & 7 (NEW) & 22.7859 & 1.9857 & 0.7501 & 0.3729 & 0.1180 \\
\hline Vapor & ST6B & 4 (NEW) & 22.6566 & 2.0017 & 0.7558 & 0.3701 & 0.1210 \\
\hline Vapor & ST6 & 5 (NEW) & 24.1676 & 2.0248 & 0.7529 & 0.3762 & 0.1298 \\
\hline Vapor & HA25W & 8 (IDMS) & 11.4579 & 1.0080 & 0.7700 & 0.3710 & 0.1170 \\
\hline Vapor & CW7MW & 6 (NEW) & 24.1838 & 1.9892 & 0.7527 & 0.3751 & 0.1284 \\
\hline Vapor & ALUMINA 203 & 1 (NEW) & 12.8934 & 1.9737 & 0.7984 & 0.3766 & 0.1434 \\
\hline Vapor & ULTIMET & 1 (NEW) & 23.8085 & 1.6622 & 0.8731 & 0.3758 & 0.1394 \\
\hline Liquid/Napor & HC22WW & 9 (NEW) & 23.1567 & 1.9913 & 0.7556 & 0.3726 & 0.1196 \\
\hline Liquid/Napor & HC276W & 8 (NEW) & 23.7500 & 1.9935 & 0.7505 & 0.3729 & 0.1211 \\
\hline Liquid/Napor & ST6B & 5 (NEW) & 22.4580 & 1.9981 & 0.7532 & 0.3694 & 0.1208 \\
\hline Liquid/Napor & ST6 & 6 (NEW) & 24.0479 & 2.0109 & 0.7506 & 0.3705 & 0.1319 \\
\hline Liquid/Napor & HA25W & 9 (IDMS) & 11.6096 & 1.0090 & 0.7690 & 0.3720 & 0.1200 \\
\hline Liquid/Napor & CW7MW & 7 (NEW) & 23.4491 & 1.9946 & 0.7527 & 0.3762 & 0.1258 \\
\hline Liquid/Napor & ALUMINA 203 & 2 (NEW) & 12.4921 & 1.9648 & 0.7801 & 0.3793 & 0.1396 \\
\hline Llquid/Napor & ULTIMET & 2 (NEW) & 21.8920 & 1.5209 & 0.8710 & 0.3750 & 0.1358 \\
\hline
\end{tabular}

* $W=$ Welded Coupon

* $($ New $)=$ Coupons purchased May, 1993 and marked with alloy, heat no. and coupon number.

* $($ IDMS $)=$ Coupons purchased before 1989 and marked with alloy, coupon number and IDMS.

VAPQR

(TOP)
LIQUID/VAPQR

(BDTTOM)

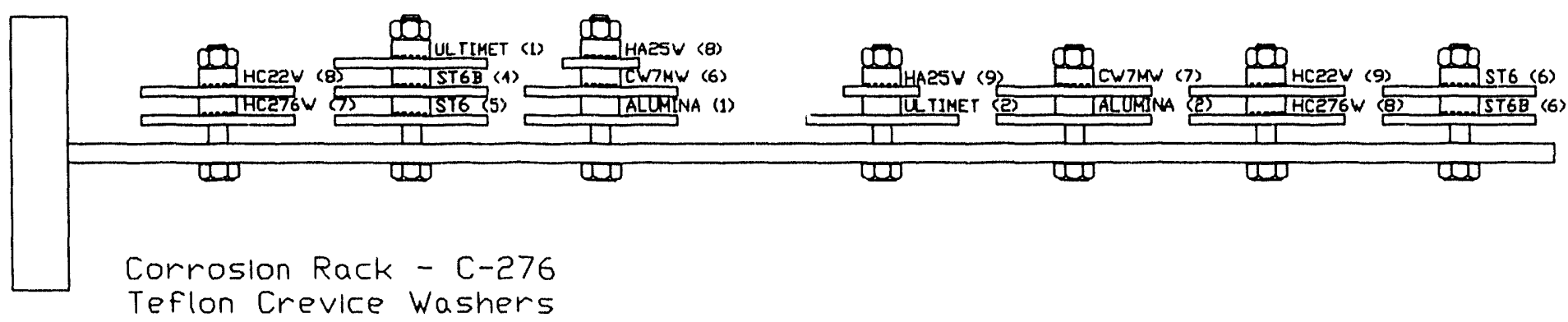


J. R. Zamecnik

October 4, 1992

Page 7 of 18

WSRC-TR-93-518

Table 3

SRAT/SME Condenser

\begin{tabular}{|c|c|c|c|c|c|c|c|}
\hline $\begin{array}{c}\text { Coupon } \\
\text { Location }\end{array}$ & $\begin{array}{c}\text { Material } \\
\text { Type }\end{array}$ & $\begin{array}{c}\text { Coupon } \\
\text { No. }\end{array}$ & $\begin{array}{c}\text { Weight } \\
\text { (g) }\end{array}$ & $\begin{array}{c}\text { Length } \\
\text { (in) }\end{array}$ & $\begin{array}{c}\text { Width } \\
\text { (in) }\end{array}$ & $\begin{array}{c}\text { Hole Dia. } \\
\text { (in) }\end{array}$ & $\begin{array}{c}\text { Thickness } \\
\text { (in) }\end{array}$ \\
\hline- & HG3W (U) & 2 (IDMS) & 32.7324 & 3.2200 & 0.7590 & 0.3840 & 0.1180 \\
\hline- & $316 L W(U)$ & 3 (IDMS) & 28.9368 & 3.2200 & 0.7570 & 0.3860 & 0.1110 \\
\hline- & ALEXNW (U) & 7 (IDMS) & 30.3433 & 3.2200 & 0.7540 & 0.3870 & 0.1130 \\
\hline- & $317 L W(\mathrm{U})$ & 7 (IDMS) & 24.5447 & 3.1250 & 0.7620 & 0.3890 & 0.0930 \\
\hline- & HC22W & 2 (NEW) & 23.4248 & 1.9943 & 0.7537 & 0.3730 & 0.1191 \\
\hline- & HC276W & 4 (IDMS) & 9.8974 & 1.0060 & 0.7760 & 0.3886 & 0.1050 \\
\hline
\end{tabular}

- $(U)=$ Stressed U-Bend Coupon

* $W=$ Welded Coupon

* $($ IDMS $)=$ Coupons purchased before 1989 and marked with alloy, coupon number and IDMS.

* $(\mathrm{New})=$ Coupons purchased May, 1993 and marked with alloy, heat no. and coupon number.

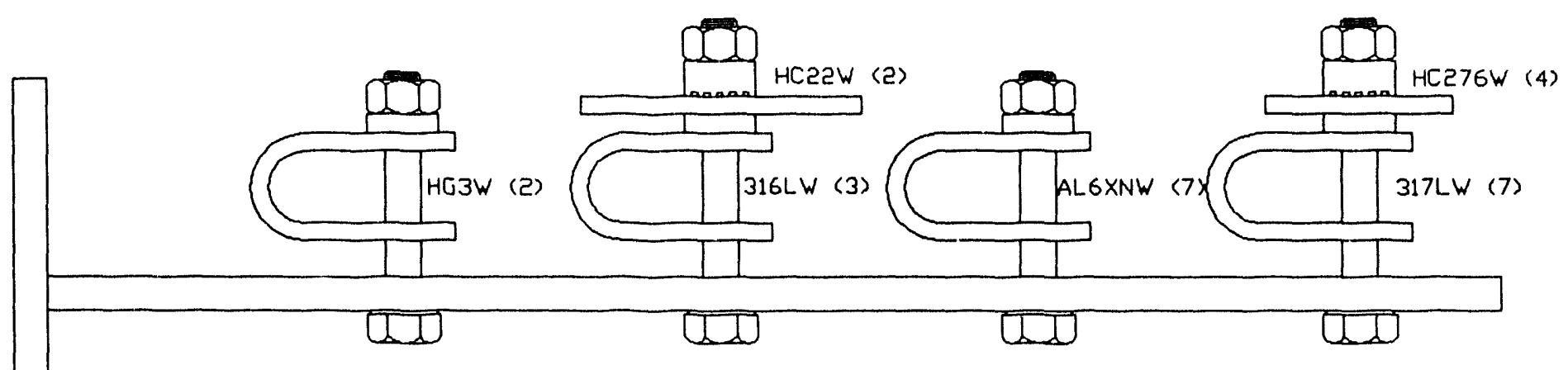

Corrosion Rack - C-276

Teflon Crevice Washers 
J. R. Zamecnik

October 4, 1992

Page 8 of 18

WSRC-TR-93-518

Table 4

Mercury Water Wash Tank - Vapor space

\begin{tabular}{|c|c|c|c|c|c|c|c|}
\hline $\begin{array}{c}\text { Coupon } \\
\text { Location }\end{array}$ & $\begin{array}{c}\text { Material } \\
\text { Type }\end{array}$ & $\begin{array}{c}\text { Coupon } \\
\text { No. }\end{array}$ & $\begin{array}{c}\text { Weight } \\
\text { (g) }\end{array}$ & $\begin{array}{c}\text { Length } \\
\text { (in) }\end{array}$ & $\begin{array}{c}\text { Width } \\
(\mathrm{in})\end{array}$ & $\begin{array}{c}\text { Hole Dia. } \\
\text { (in) }\end{array}$ & $\begin{array}{c}\text { Thickness } \\
\text { (in) }\end{array}$ \\
\hline- & $316 \mathrm{LW}$ & 1 (NEW) & 19.9994 & 1.9968 & 0.7557 & 0.3712 & 0.1141 \\
\hline- & $304 \mathrm{LW}$ & 1 (NEW) & 19.3236 & 1.9971 & 0.7553 & 0.3712 & 0.1082 \\
\hline- & ALEXNW & 9 (IDMS) & 20.6441 & 2.0000 & 0.7630 & 0.3690 & 0.1140 \\
\hline- & HC276W & 4 (NEW) & 23.8781 & 1.9907 & 0.7545 & 0.3737 & 0.1207 \\
\hline- & ST6B & 1 (NEW) & 22.7116 & 2.0012 & 0.7543 & 0.3692 & 0.1220 \\
\hline
\end{tabular}

- $W=$ Welded Coupon

* (IDMS) $=$ Coupons purchased before 1989 and marked with alloy, coupon number and IDMS.

* $(\mathrm{New})=$ Coupons purchased May, 1993 and marked with alloy, heat no. and coupon number.

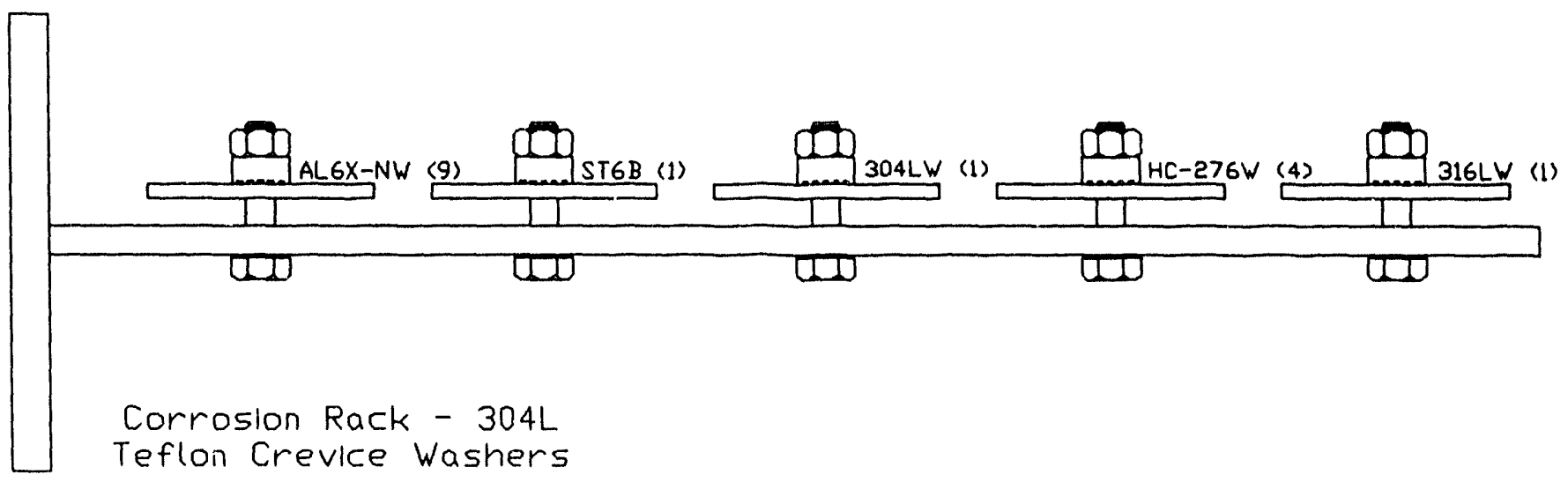


J. R. Zamecnik

October 4, 1992

Page 9 of 18

WSRC-TR-93-518

Table 5

Melter Feed Tank - Vapor Space and Liquid/vapor zone

\begin{tabular}{|c|c|c|c|c|c|c|c|c|}
\hline $\begin{array}{c}\text { Coupon } \\
\text { Location }\end{array}$ & $\begin{array}{c}\text { Material } \\
\text { Type }\end{array}$ & $\begin{array}{c}\text { Coupon } \\
\text { No. }\end{array}$ & $\begin{array}{c}\text { Weight } \\
(\mathrm{g})\end{array}$ & $\begin{array}{c}\text { Length } \\
\text { (in) }\end{array}$ & $\begin{array}{c}\text { Width } \\
\text { (in) }\end{array}$ & $\begin{array}{c}\text { Hole Dia. } \\
\text { (in) }\end{array}$ & $\begin{array}{c}\text { Thickness } \\
\text { (in) }\end{array}$ \\
\hline Vapor & HC:2W & 6 (NEW) & 23.1114 & 1.9940 & 0.7518 & 0.3705 & 0.1166 \\
\hline Vapor & HC276W & 5 (NEW) & 23.1190 & 1.9913 & 0.7539 & 0.3739 & 0.1210 \\
\hline Vapor & ST6B & 3 (NEW) & 22.5683 & 2.0044 & 0.7545 & 0.3684 & 0.1210 \\
\hline Vapor & ST6 & 7 (NEW) & 25.3211 & 2.0229 & 0.7505 & 0.3755 & 0.1335 \\
\hline Vapor & HA25W & 7 (IDMS) & 11.2788 & 1.0090 & 0.7730 & 0.3770 & 0.1170 \\
\hline Vapor & CW7MW & 4 (NEW) & 23.9968 & 1.9855 & 0.7545 & 0.3749 & 0.1296 \\
\hline Vapor & ALUMINA 203 & 3 (NEW) & 12.4202 & 1.9597 & 0.7950 & 0.3787 & 0.1411 \\
\hline Liquid/Vapor & HC22W & 7 (NEW) & 23.0969 & 1.9854 & 0.7497 & 0.3735 & 0.1230 \\
\hline Liquid/Napor & HC276W & 6 (NEW) & 22.9075 & 1.9895 & 0.7507 & 0.3724 & 0.1185 \\
\hline Liquid/Napor & ST6B & 2 (NEW) & 22.6839 & 2.0024 & 0.7561 & 0.3688 & 0.1212 \\
\hline Liquid/Napor & ST6 & 2 (NEW) & 22.9679 & 2.0057 & 0.7509 & 0.3769 & 0.1240 \\
\hline Liquid/Napor & HA25W & 6 (IDMS) & 10.7947 & 0.9960 & 0.7530 & 0.3760 & 0.1170 \\
\hline Liquid/Napor & CW7MW & 5 (NEW) & 24.6706 & 1.9913 & 0.7525 & 0.3746 & 0.1321 \\
\hline Liquid/Napor & ALUMINA 203 & 4 (NEW) & 12.8279 & 1.9302 & 0.7864 & 0.3788 & 0.1408 \\
\hline
\end{tabular}

- $W=$ Welded Coupon

* $($ IDMS $)=$ Coupons purchased before 1989 and marked with alloy, coupon number and IDMS.

- $($ New $)=$ Coupons purchased May, 1993 and marked with alloy, heat no. and coupon number.

VAPDR

(TDP)
LIQIID/VAPOR

(BDTTOM)

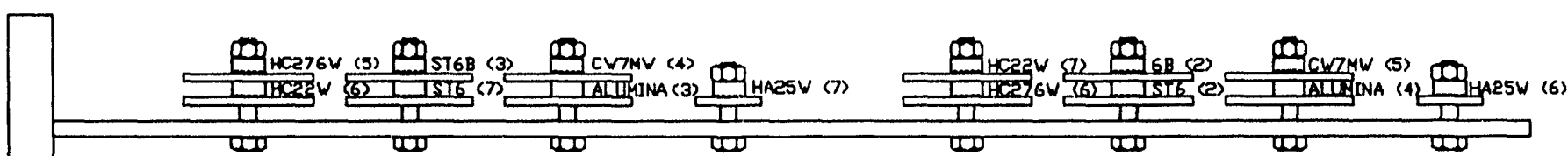

Corrosion Rack - C-276

Teflon Crevice Washers 
J. R. Zamecnik

October 4,1992

Page 10 of 18

WSRC-TR-93-518

Table 6

Quencher - Inlet

\begin{tabular}{|c|c|c|c|c|c|c|c|}
\hline $\begin{array}{c}\text { Coupon } \\
\text { Location }\end{array}$ & $\begin{array}{c}\text { Material } \\
\text { Type }\end{array}$ & $\begin{array}{c}\text { Coupon } \\
\text { No. }\end{array}$ & $\begin{array}{c}\text { Weight } \\
(\mathrm{g})\end{array}$ & $\begin{array}{c}\text { Length } \\
\text { (in) }\end{array}$ & $\begin{array}{c}\text { Width } \\
\text { (in) }\end{array}$ & $\begin{array}{c}\text { Hole Dia. } \\
\text { (in) }\end{array}$ & $\begin{array}{c}\text { Thickness } \\
\text { (in) }\end{array}$ \\
\hline Inlet & HA556 & 1 (NEW) & 9.0770 & 1.0048 & 0.7529 & 0.3745 & 0.1064 \\
\hline Inlet & $1625 W$ & 3 (IDMS) & 21.5344 & 2.0150 & 0.7650 & 0.3860 & 0.1120 \\
\hline Inlet & CW7MW & 1 (NEW) & 24.2023 & 1.9902 & 0.7477 & 0.3747 & 0.1295 \\
\hline Inlet & HC22W & 3 (NEW) & 23.4605 & 1.9930 & 0.7521 & 0.3740 & 0.1218 \\
\hline Inlet & 1671 & 3 (OLD NM) & 10.5069 & 1.9410 & 0.7420 & 0.3750 & 0.0610 \\
\hline Inlet & I690W & 1 (IDMS) & 19.5600 & 2.0120 & 0.7540 & 0.3880 & 0.1080 \\
\hline Inlet & ALLORRW & 7 (IDMS) & 15.1856 & 1.9980 & 0.7660 & 0.3870 & 0.0820 \\
\hline Inlet & HC276W & 11 (NEW) & 23.6552 & 1.9875 & 0.7540 & 0.3729 & 0.1211 \\
\hline Inlet & $6 B$ COC & 1 (OLD NM) & 11.9407 & 1.5020 & 0.7540 & 0.3750 & 0.0920 \\
\hline Inlet & 8727 & 1 (OLD NM) & 12.5607 & 1.5010 & 0.7600 & 0.3700 & 0.0980 \\
\hline
\end{tabular}

* $W=$ Welded Coupon

* $($ IDMS $)=$ Coupons purchased before 1989 and marked with alloy, coupon number and IDMS.

* (OLD NM) = Coupons purchased in 1992 and marked with only alloy and coupon numbers.

* $($ New $)=$ Coupons purchased May, 1993 and marked with alloy, heat no. and coupon number.

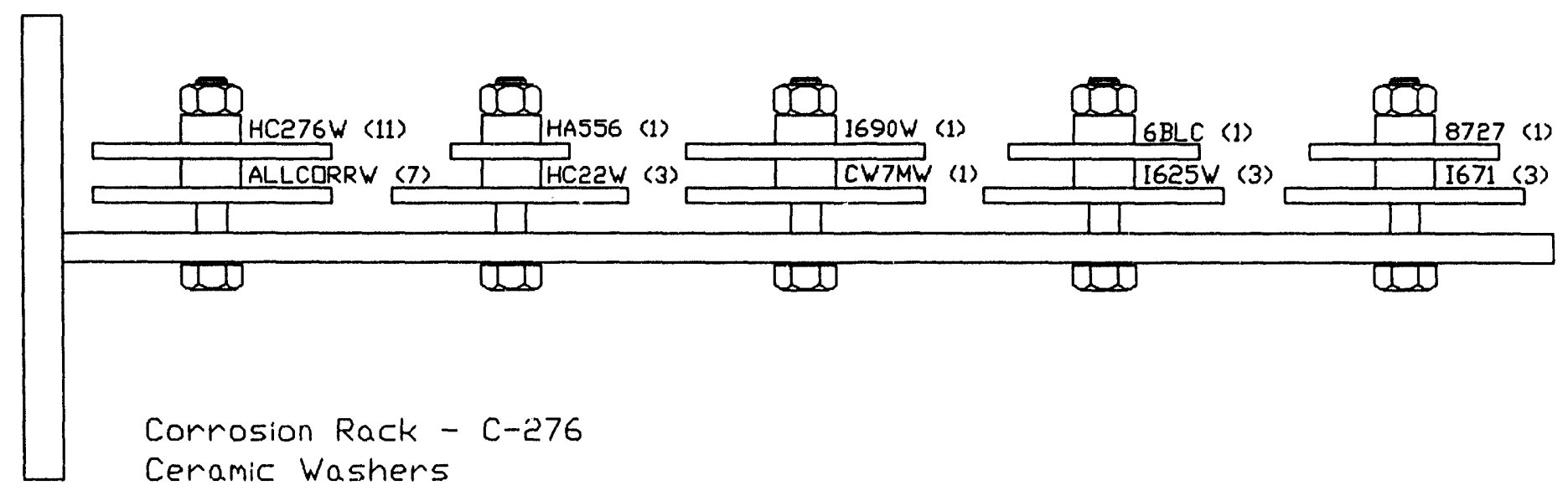


J. R. Zamecnik

October 4, 1992

Page 11 of 18

WSRC-TR-93-518

Table 7

Quencher - outlet

\begin{tabular}{|c|c|c|c|c|c|c|c|}
\hline $\begin{array}{c}\text { Coupon } \\
\text { Location }\end{array}$ & $\begin{array}{c}\text { Material } \\
\text { Type }\end{array}$ & $\begin{array}{c}\text { Coupon } \\
\text { No. }\end{array}$ & $\begin{array}{c}\text { Weight } \\
\text { (g) }\end{array}$ & $\begin{array}{c}\text { Length } \\
\text { (in) }\end{array}$ & $\begin{array}{c}\text { Width } \\
\text { (in) }\end{array}$ & $\begin{array}{c}\text { Hole Dia. } \\
\text { (in) }\end{array}$ & $\begin{array}{c}\text { Thickness } \\
\text { (in) }\end{array}$ \\
\hline Outlet & ALLCORRW & 5 (IDMS) & 16.1070 & 1.9950 & 0.7690 & 0.3890 & 0.0860 \\
\hline Outlet & ALLCORRW & 6 (IDMS) & 17.2929 & 2.0020 & 0.7690 & 0.3860 & 0.0910 \\
\hline Outlet & HC276W & 2 (NEW) & 23.9691 & 1.9930 & 0.7529 & 0.3732 & 0.1192 \\
\hline Outlet & HC276W & 3 (NEW) & 23.7823 & 1.9889 & 0.7506 & 0.3739 & 0.1207 \\
\hline Outlet & HC22W & 4 (NEW) & 23.2729 & 1.9904 & 0.7548 & 0.3736 & 0.1205 \\
\hline Outlet & HC22W & 5 (NEW) & 22.9624 & 1.9905 & 0.7527 & 0.3728 & 0.1228 \\
\hline Outlet & $1625 W$ & 4 (IDMS) & 21.0766 & 2.0070 & 0.7570 & 0.3860 & 0.1100 \\
\hline Outlet & $1625 W$ & 5 (IDMS) & 21.6151 & 2.0150 & 0.7680 & 0.3870 & 0.1120 \\
\hline Outlet & CW7MW & 2 (NEW) & 24.9789 & 1.9952 & 0.7543 & 0.3738 & 0.1324 \\
\hline Outlet & CW7MW & 3 (NEW) & 23.9800 & 1.9981 & 0.7558 & 0.3752 & 0.1290 \\
\hline
\end{tabular}

* $W=$ Welded Coupon

- $($ IDMS $)=$ Coupons purchased before 1989 and marked with alloy, coupon number and IDMS.

* $(\mathrm{New})=$ Coupons purchased May, 1993 and marked with alloy, heat no. and coupon number.

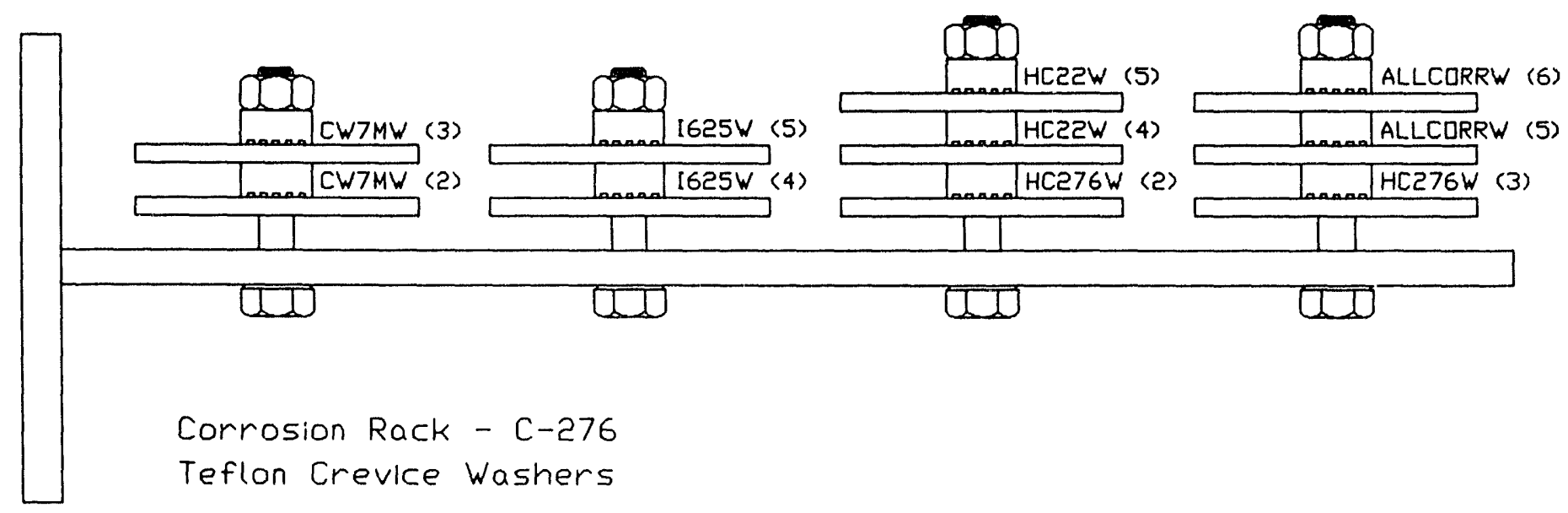


J. R. Zamecnik

October 4, 1992

Page 12 of 18

WSRC-TR-93-518

Table 8

off-Gas Condensate Tank - Vapor space and Liquid zone

\begin{tabular}{|c|c|c|c|c|c|c|c|}
\hline $\begin{array}{c}\text { Coupon } \\
\text { Location }\end{array}$ & $\begin{array}{c}\text { Material } \\
\text { Type }\end{array}$ & $\begin{array}{c}\text { Coupon } \\
\text { No. }\end{array}$ & $\begin{array}{c}\text { Weight } \\
(\mathbf{g})\end{array}$ & $\begin{array}{c}\text { Length } \\
\text { (in) }\end{array}$ & $\begin{array}{c}\text { Width } \\
\text { (in) }\end{array}$ & $\begin{array}{c}\text { Hole Dia. } \\
\text { (in) }\end{array}$ & $\begin{array}{c}\text { Thickness } \\
\text { (in) }\end{array}$ \\
\hline Vapor & HC22W & 10 (NEW) & 22.9969 & 1.9923 & 0.7534 & 0.3735 & 0.1215 \\
\hline Vapor & HC276W & 9 (NEW) & 23.9352 & 1.9910 & 0.7513 & 0.3720 & 0.1226 \\
\hline Vapor & CW7MW & 8 (NEW) & 24.2796 & 1.9930 & 0.7528 & 0.3761 & 0.1292 \\
\hline Vapor & ST6 & 3 (NEW) & 22.4380 & 1.9787 & 0.7526 & 0.3768 & 0.1237 \\
\hline Vapor & HA25W & 7 (IDMS) & 24.6412 & 1.9960 & 0.7410 & 0.3840 & 0.1220 \\
\hline Vapor & ST6B & 6 (NEW) & 22.5364 & 2.0033 & 0.7554 & 0.3692 & 0.1217 \\
\hline Liquid & HC22W & 11 (NEW) & 22.9261 & 1.9878 & 0.7560 & 0.3733 & 0.1208 \\
\hline Liquild & HC276W & 10 (NEW) & 23.4338 & 1.9960 & 0.7542 & 0.3720 & 0.1191 \\
\hline Liquid & CW7MW & 9 (NEW) & 23.3633 & 1.9957 & 0.7502 & 0.3757 & 0.1239 \\
\hline Llquid & ST6 & 4 (NEW) & 24.6089 & 2.0137 & 0.7525 & 0.3770 & 0.1314 \\
\hline Liquid & HA25W & 8 (IDMS) & 24.4678 & 1.9730 & 0.7530 & 0.3820 & 0.1220 \\
\hline Llquid & ST6B & 7 (NEW) & 22.5504 & 2.0000 & 0.7544 & 0.3724 & 0.1213 \\
\hline
\end{tabular}

* $W=$ Welded Coupon

- $($ IDMS $)=$ Coupons purchased before 1989 and marked with alloy, coupon number and IDMS.

- $(\mathrm{New})=$ Coupons purchased May, 1993 and marked with alloy, heat no. and coupon number.

VAPQR

(TOP)

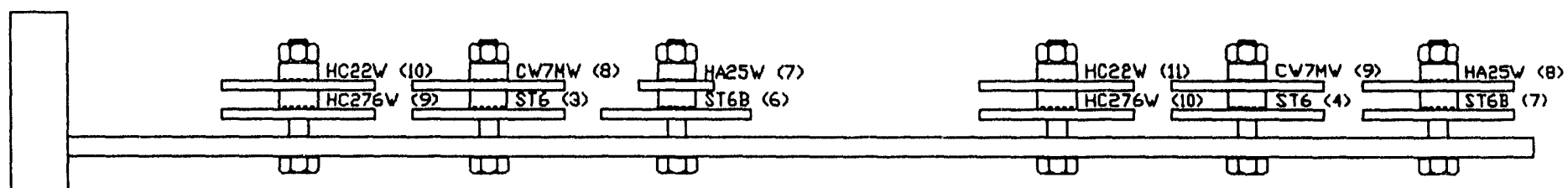

LIQUID

(BDTTOM)

Corrosion Rack - C-276

Teflon Crevice Washers 
J. R. Zamecnik

October 4, 1992

Page 13 of 18

WSRC-T'R-93-518

Table 9

OffuGas Condenser - Vapor Inlet

\begin{tabular}{|c|c|c|c|c|c|c|c|}
\hline $\begin{array}{c}\text { Coupon } \\
\text { Location }\end{array}$ & $\begin{array}{c}\text { Material } \\
\text { Type }\end{array}$ & $\begin{array}{c}\text { Coupon } \\
\text { No. }\end{array}$ & $\begin{array}{c}\text { Weight } \\
(\mathrm{g})\end{array}$ & $\begin{array}{c}\text { Length } \\
\text { (in) }\end{array}$ & $\begin{array}{c}\text { Width } \\
\text { (in) }\end{array}$ & $\begin{array}{c}\text { Hole Dia. } \\
\text { (in) }\end{array}$ & $\begin{array}{c}\text { Thickness } \\
\text { (in) }\end{array}$ \\
\hline Inlet & HC276W & 18 (IDMS) & 21.6739 & 2.0000 & 0.7630 & 0.3910 & 0.1100 \\
\hline Inlet & 317 WW (U) & 6 (IDMS) & 24.3400 & 3.125 & 0.725 & 0.389 & 0.093 \\
\hline Inlet & HC22W & 15 (IDMS) & 21.4880 & 1.9830 & 0.7570 & 0.3910 & 0.1140 \\
\hline Inlet & AL6XNW (U) & 6 (IDMS) & 30.2042 & 3.2100 & 0.7520 & 0.3880 & 0.1160 \\
\hline
\end{tabular}

* $(U)=$ Stressed U-Bend Coupon

* $W=$ Welded Coupon

* $($ IDMS $)=$ Coupons purchased before 1989 and marked with alloy, coupon number and IDMS.

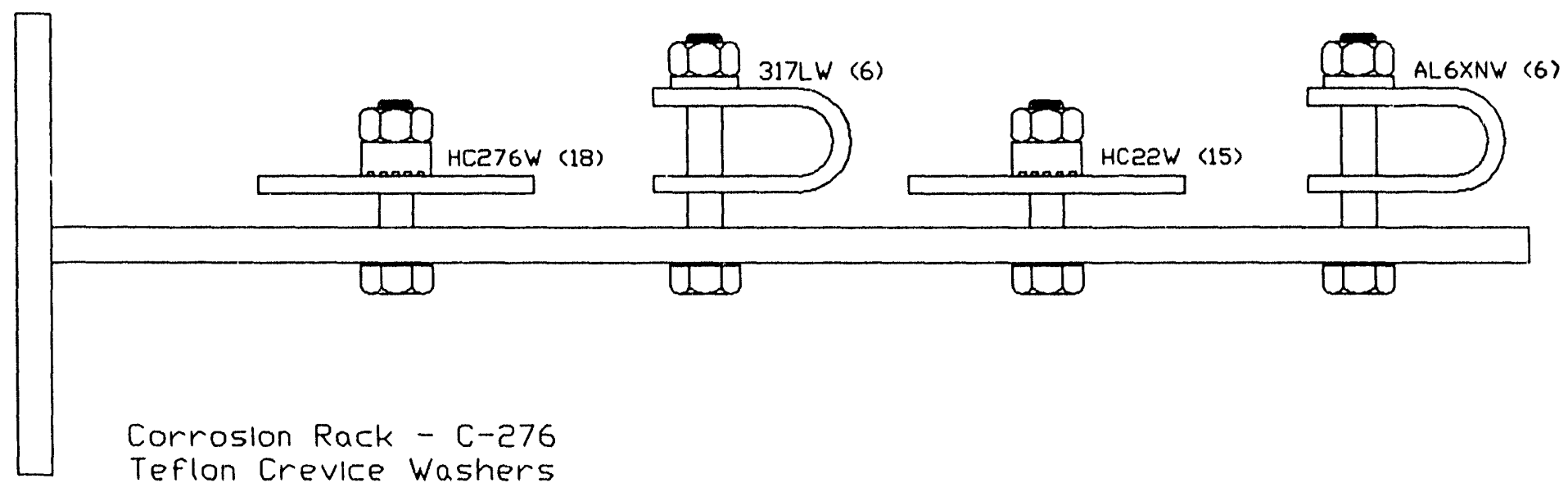


J. R. Zamecnik

October 4, 1992

Page 14 of 18

WSRC-TR-93-518

Table 10

Off-Gas Condenser - Drain

\begin{tabular}{|c|c|c|c|c|c|c|c|}
\hline $\begin{array}{c}\text { Coupon } \\
\text { Location }\end{array}$ & $\begin{array}{c}\text { Material } \\
\text { Type }\end{array}$ & $\begin{array}{c}\text { Coupon } \\
\text { No. }\end{array}$ & $\begin{array}{c}\text { Weight } \\
\text { (g) }\end{array}$ & $\begin{array}{c}\text { Length } \\
\text { (in) }\end{array}$ & $\begin{array}{c}\text { Width } \\
\text { (in) }\end{array}$ & $\begin{array}{c}\text { Hole Dia. } \\
\text { (in) }\end{array}$ & $\begin{array}{c}\text { Thickness } \\
\text { (in) }\end{array}$ \\
\hline Drain & HC276W & 16 (IDMS) & 21.7313 & 1.9960 & 0.7700 & 0.3890 & 0.1120 \\
\hline Drain & $317 L W($ (U) & 5 (IDMS) & 23.9616 & 3.1250 & 0.7590 & 0.3900 & 0.0910 \\
\hline Drain & HC22W & 11 (IDMS) & 22.3514 & 2.0010 & 0.7640 & 0.3880 & 0.1160 \\
\hline Drain & ALEXNW (U) & 5 (IDMS) & 30.7225 & 3.2200 & 0.7570 & 0.3840 & 0.1180 \\
\hline
\end{tabular}

* $(U)=$ Stressed $U$-Bend Coupon

* $W=$ Welded Coupon

- $($ IDMS $)=$ Coupons purchased before 1989 and marked with alloy, coupon number and IDMS.

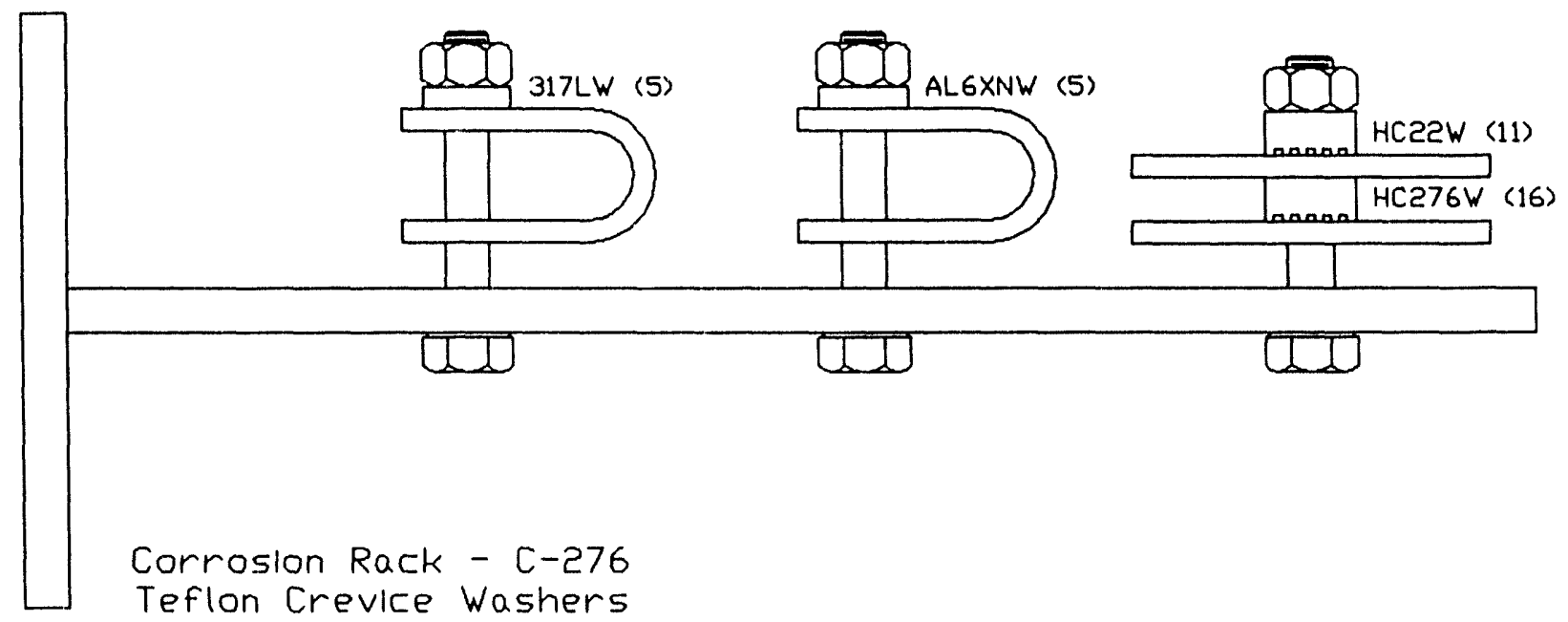


J. R. Zamecnik

October 4,1992

Page 15 of 18

WSRC-TR-93-518

Table 11

steam Atomized Bcrubber - Drain Line

\begin{tabular}{|c|c|c|c|c|c|c|c|}
\hline $\begin{array}{c}\text { Coupon } \\
\text { Location }\end{array}$ & $\begin{array}{c}\text { Material } \\
\text { Type }\end{array}$ & $\begin{array}{c}\text { Coupon } \\
\text { No. }\end{array}$ & $\begin{array}{c}\text { Weight } \\
\text { (g) }\end{array}$ & $\begin{array}{c}\text { Length } \\
\text { (in) }\end{array}$ & $\begin{array}{c}\text { Width } \\
\text { (in) }\end{array}$ & $\begin{array}{c}\text { Hole Dia. } \\
\text { (in) }\end{array}$ & $\begin{array}{c}\text { Thickness } \\
\text { (in) }\end{array}$ \\
\hline- & ST6 & 1 (NEW) & 21.1700 & 1.9741 & 0.7524 & 0.3771 & $0.117 y$ \\
\hline- & ALLCORRW & 4 (IDMS) & 15.5776 & 2.0030 & 0.7680 & 0.3880 & 0.0850 \\
\hline- & $6 B$ & 8 (OLD NM) & 18.1784 & 1.4980 & 0.7540 & 0.3700 & 0.1310 \\
\hline- & HC22W & 1 (NEW) & 23.7065 & 2.0030 & 0.7550 & 0.3739 & 0.1231 \\
\hline- & HC276W & 2 (IDMS) & 9.5544 & 1.0100 & 0.7680 & 0.3880 & 0.1020 \\
\hline
\end{tabular}

* $W=$ Welded Coupon

* $($ IDMS $)=$ Coupons purchased before 1989 and marked with alloy, coupon number and IDMS.

- $(O L D N M)=$ Coupons purchased in 1992 and marked with only alloy and coupon numbers.

* $(\mathrm{New})=$ Coupons purchased May, 1993 and marked with alloy, heat no. and coupon number.

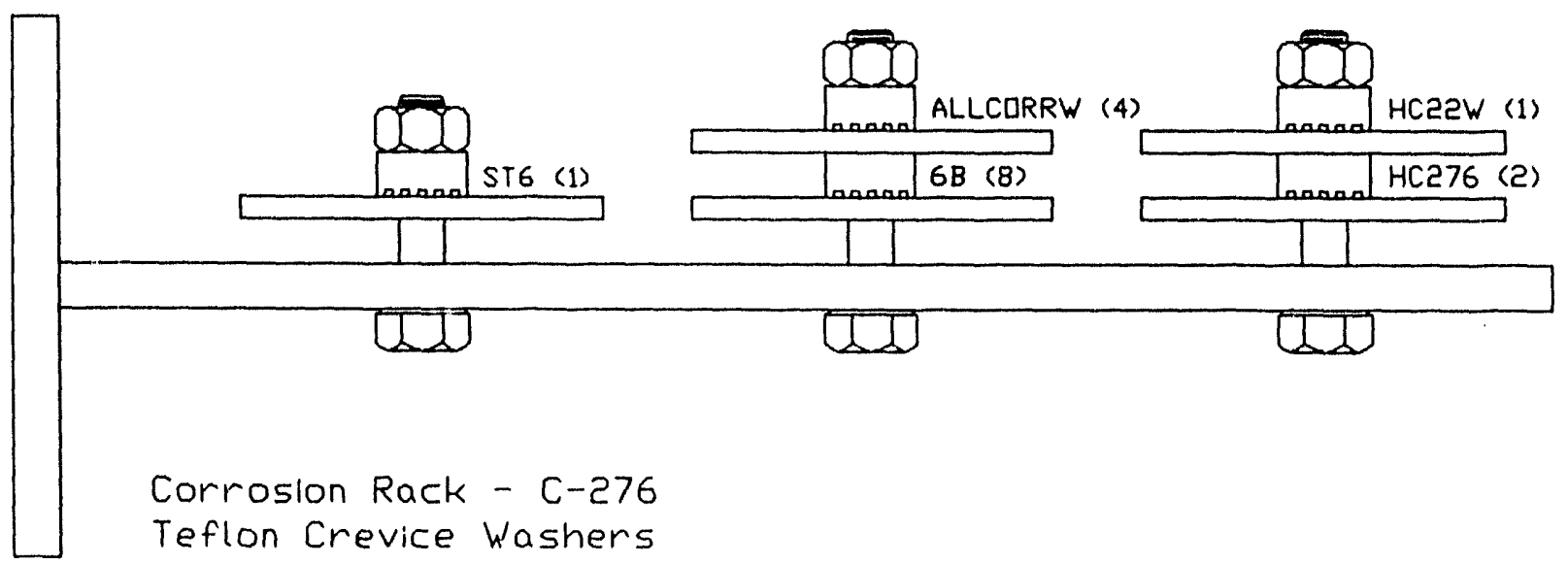


J. R. Zamecnik

October 4, 1992

Page 16 of 18

WSRC-TR-93-518

Table 12

High Efficienoy Mist Eliminator - Inlet

\begin{tabular}{|c|c|c|c|c|c|c|c|}
\hline $\begin{array}{c}\text { Coupon } \\
\text { Location }\end{array}$ & $\begin{array}{c}\text { Material } \\
\text { Type }\end{array}$ & $\begin{array}{c}\text { Coupon } \\
\text { No. }\end{array}$ & $\begin{array}{c}\text { Weight } \\
\text { (g) }\end{array}$ & $\begin{array}{c}\text { Length } \\
\text { (in) }\end{array}$ & $\begin{array}{c}\text { Width } \\
\text { (in) }\end{array}$ & $\begin{array}{c}\text { Hole Dia. } \\
\text { (in) }\end{array}$ & $\begin{array}{c}\text { Thickness } \\
\text { (in) }\end{array}$ \\
\hline Inlet & AL6XNW & 8 (IDMS) & 19.8633 & 2.0010 & 0.7650 & 0.3860 & 0.1140 \\
\hline Inlet & HC276W & 1 (NEW) & 23.2942 & 1.9912 & 0.7517 & 0.3737 & 0.1199 \\
\hline Inlet & $316 L W(U)$ & $4($ IDMS) & 29.1366 & 3.2250 & 0.7540 & 0.3890 & 0.1110 \\
\hline Inlet & $304 \mathrm{LW}(\mathrm{U})$ & $3(\mathrm{IDMS})$ & 29.1017 & 3.2200 & 0.7610 & 0.3890 & 0.1120 \\
\hline
\end{tabular}

* $(U)=$ Stressed $U-B e n d$ Coupon

* $W=$ Welded Coupon

- $($ IDMS $)=$ Coupons purchased before 1989 and marked with alloy, coupon number and IDMS.

* $($ New $)=$ Coupons purchased May, 1993 and marked with alloy, heat no. and coupon number.

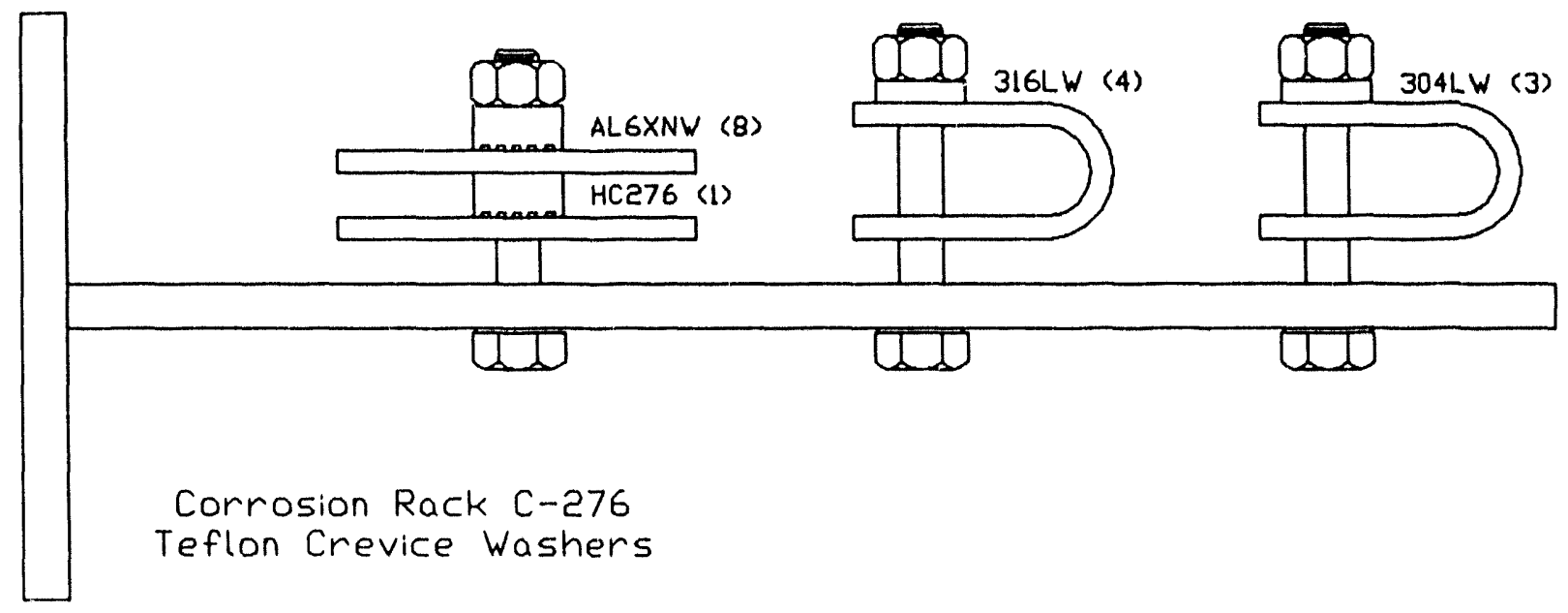


J. R. Zamecnik

October 4, 1992

Page 17 of 18

WSRC-TR-93-518

Table 13

High Effioienoy Mist Eliminator - outlet

\begin{tabular}{|c|c|c|c|c|c|c|c|}
\hline $\begin{array}{l}\text { Coupon } \\
\text { Location }\end{array}$ & $\begin{array}{c}\text { Material } \\
\text { Type }\end{array}$ & $\begin{array}{c}\text { Coupon } \\
\text { No. }\end{array}$ & $\begin{array}{l}\text { Weight } \\
\text { (g) }\end{array}$ & $\begin{array}{l}\text { Length } \\
\text { (in) }\end{array}$ & $\begin{array}{l}\text { Width } \\
\text { (in) }\end{array}$ & $\begin{array}{l}\text { Hole Dia. } \\
\text { (in) }\end{array}$ & $\begin{array}{l}\text { Thickness } \\
\text { (in) }\end{array}$ \\
\hline Outlat & $317 \mathrm{LW}$ & 3 (IDMS) & 18.7550 & 1.9930 & 0.7620 & 0.3870 & 0.1040 \\
\hline Outlet & ALGXNW & 6 (IDMS) & 20.0324 & 2.0020 & 0.7570 & 0.3890 & 0.1140 \\
\hline Outlet & $316 \mathrm{LW}$ & 2 (NEW) & 19.6844 & 1.8946 & 0.7550 & 0.3714 & 0.1123 \\
\hline Outlet & $304 \mathrm{LW}$ & 2 (NEW) & 19.4729 & 1.9952 & 0.7552 & 0.3709 & 0.1120 \\
\hline
\end{tabular}

* $W=$ Welded Coupon

- $($ IDMS $)=$ Coupons purchased before 1989 and marked with alloy, coupon number and IDMS.

- $($ New $)=$ Coupons purchased May, 1993 and marked with alloy, heat no. and coupon number.

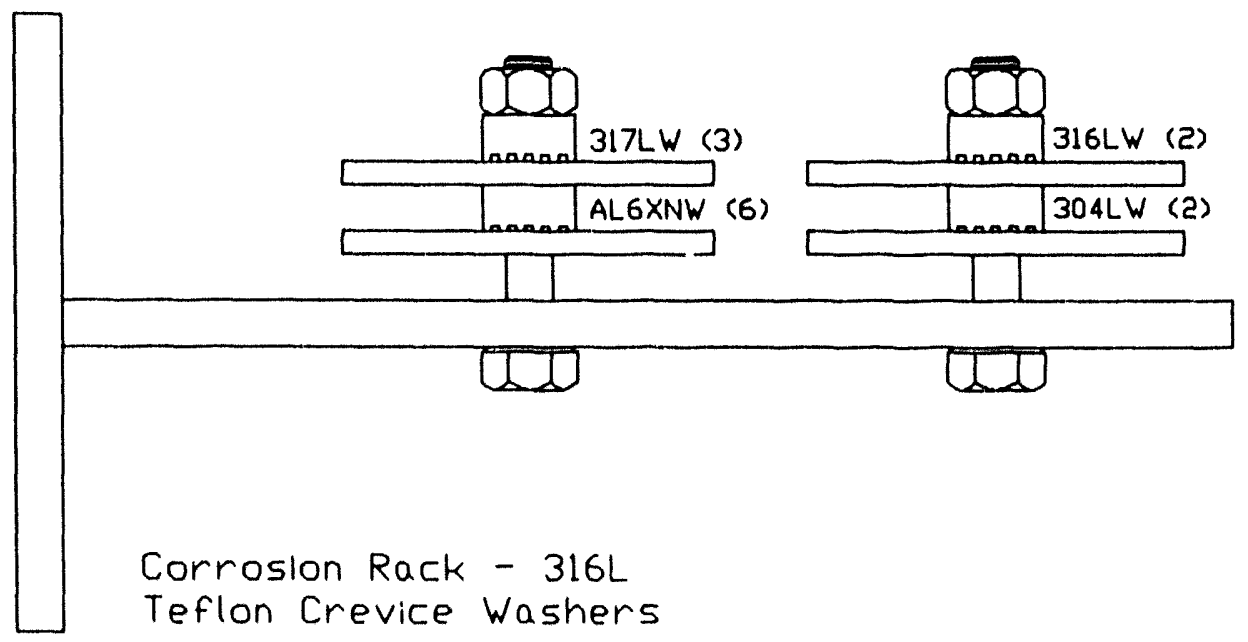


J. R. Zamecnik

October 4, 1992

Page 18 of 18

WSRC-TR-93-518

Table 14

HEPA - Inlet

\begin{tabular}{|c|c|c|c|c|c|c|c|}
\hline $\begin{array}{l}\text { Coupon } \\
\text { Location }\end{array}$ & $\begin{array}{c}\text { Material } \\
\text { Type }\end{array}$ & $\begin{array}{c}\text { Coupon } \\
\text { No. }\end{array}$ & $\begin{array}{c}\text { Woight } \\
\text { (g) }\end{array}$ & $\begin{array}{l}\text { Length } \\
\text { (in) }\end{array}$ & $\begin{array}{l}\text { Width } \\
\text { (in) }\end{array}$ & $\begin{array}{l}\text { Hole Dia. } \\
\text { (in) }\end{array}$ & $\begin{array}{l}\text { Thickness } \\
\text { (in) }\end{array}$ \\
\hline Inlet & $317 \mathrm{LW}$ & 1 (NEW) & 20.1253 & 1.9947 & 0.7577 & 0.3730 & 0.1165 \\
\hline Inlet & ALEXNW & 7 (IDMS) & 20.0355 & 2.0020 & 0.7600 & 0.3860 & 0.1130 \\
\hline Inlet & $316 L W$ & 3 (NEW) & 19.8142 & 1.9981 & 0.7503 & 0.3724 & 0.1138 \\
\hline Intet & $304 L W$ & 3 (NEW) & 18.7561 & 1.8928 & 0.7535 & 0.3722 & 0.1117 \\
\hline
\end{tabular}

- $W=$ Welded Coupon

- (IDMS) = Coupons purchased before 1989 and marked with alloy, coupon number and IDMS.

- $($ New $)=$ Coupons purchased May, 1993 and marked with alloy, heat no. and coupon number.

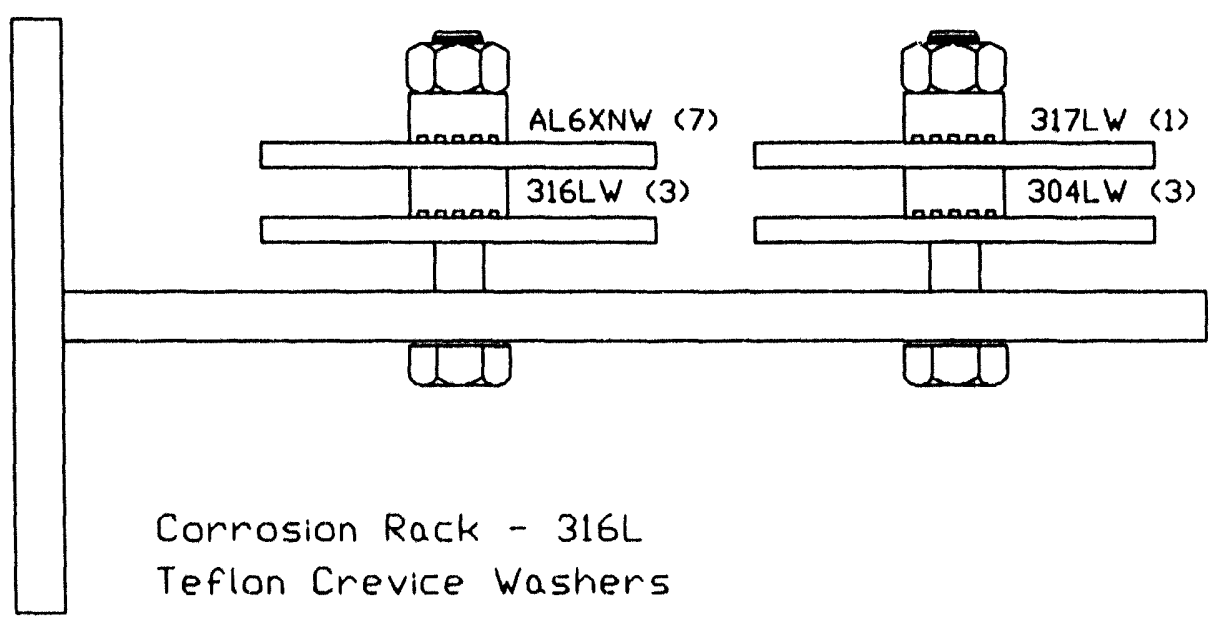



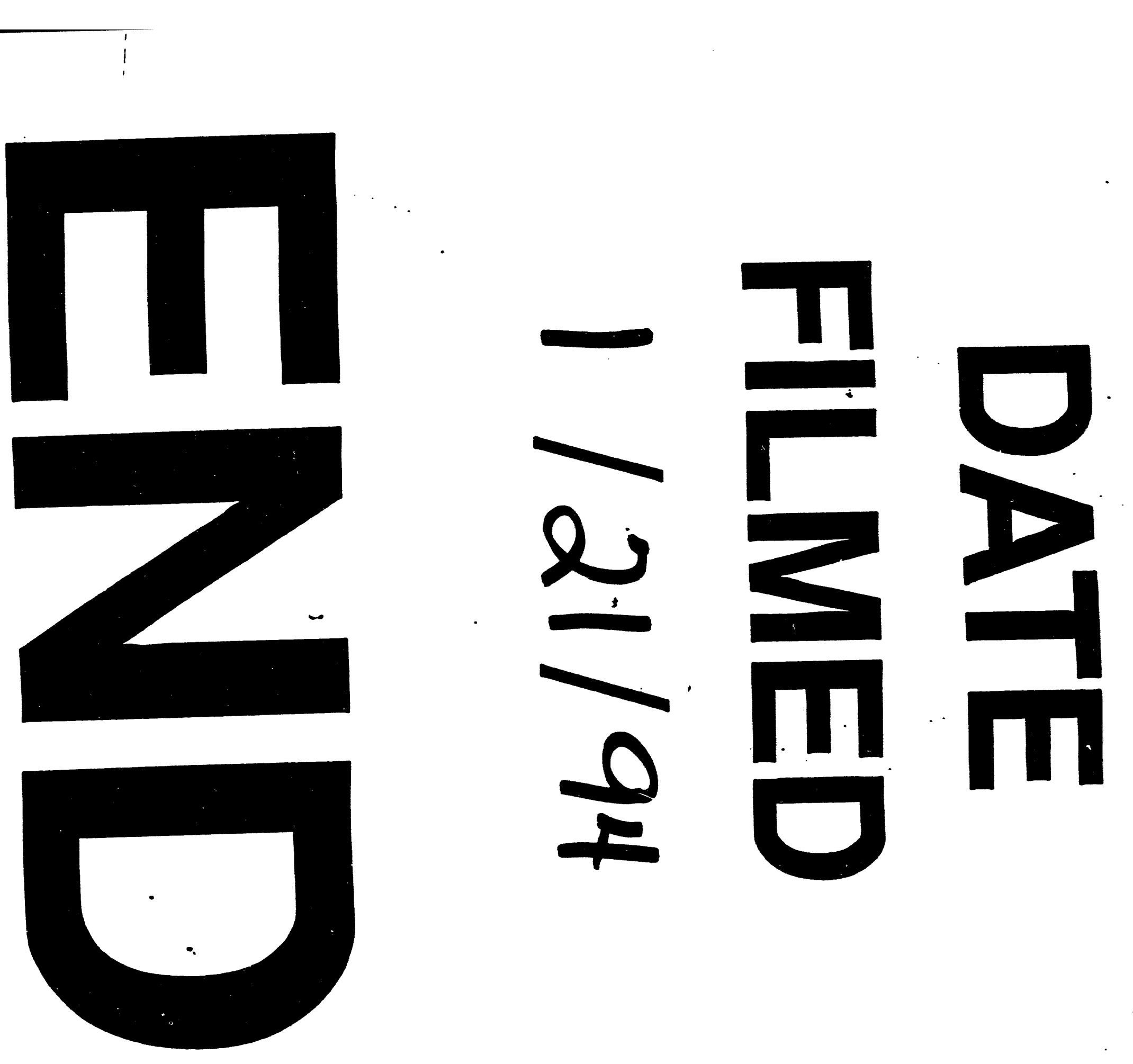


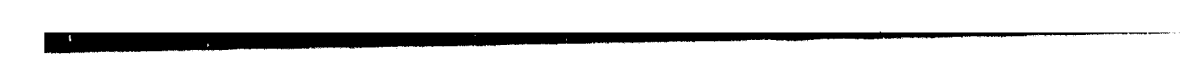

\section{$-7$}

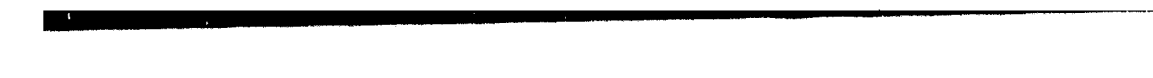

$\emptyset$

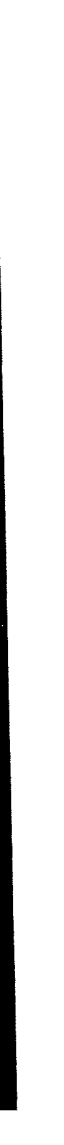

